\title{
Small-amplitude collective modes of a finite-size unitary Fermi gas in deformed traps
}

Fei, $\mathrm{Na}$

2019-11-14

Fei , N , Pei , J C , Wang , K \& Kortelainen , M 2019 , ' Small-amplitude collective modes of a finite-size unitary Fermi gas in deformed traps ' , Physical Review A, vol. 100 , no. 5 , 053613 . https://doi.org/10.1103/PhysRevA.100.053613

http://hdl.handle.net/10138/312073

https://doi.org/10.1103/PhysRevA.100.053613

publishedVersion

Downloaded from Helda, University of Helsinki institutional repository.

This is an electronic reprint of the original article.

This reprint may differ from the original in pagination and typographic detail.

Please cite the original version. 


\title{
Small-amplitude collective modes of a finite-size unitary Fermi gas in deformed traps
}

\author{
Na Fei, ${ }^{1}$ J. C. Pei, ${ }^{1, *}$ K. Wang, ${ }^{1}$ and M. Kortelainen ${ }^{2,3}$ \\ ${ }^{1}$ State Key Laboratory of Nuclear Physics and Technology, School of Physics, Peking University, Beijing 100871, China \\ ${ }^{2}$ Department of Physics, P.O. Box 35 (YFL), University of Jyvaskyla, FI-40014 Jyvaskyla, Finland \\ ${ }^{3}$ Helsinki Institute of Physics, University of Helsinki, P.O. Box 64, FI-00014 Helsinki, Finland
}

(Received 31 July 2019; published 14 November 2019)

\begin{abstract}
We have investigated collective breathing modes of a unitary Fermi gas in deformed harmonic traps. The ground state is studied by the superfluid local density approximation (SLDA) and small-amplitude collective modes are studied by the iterative quasiparticle random phase approximation (QRPA). The results illustrate the evolutions of collective modes of a small system in traps from spherical to elongated or pancake-shaped deformations. For small spherical systems, the influences of different SLDA parameters are significant, and, in particular, a large pairing strength can shift up the oscillation frequency of collective modes. The transition currents from QRPA show that the compressional flow patterns are nontrivial and dependent on the deformation. Finally, the finite-size effects are demonstrated to be reasonable when progressing towards larger systems. The hydrodynamical results of collective frequencies can be reproduced by SLDA-QRPA with reduced pairing strengths. Our studies indicate that experiments on small and medium systems are valuable for understanding effective interactions in systems with varying sizes and trap deformations.
\end{abstract}

DOI: 10.1103/PhysRevA.100.053613

\section{INTRODUCTION}

The studies of strongly interacting ultracold atomic gases have interdisciplinary interests in quantum many-body systems [1,2], such as condensed matter, nuclear physics, and neutron stars. By manipulating the $s$-wave scattering length of fermion atoms in experiments, the superfluidity phases can change smoothly from BCS to Bose-Einstein condensates (BECs). There are numerous studies of cold Fermi gases about their static and dynamic properties [3-5], demonstrating versatile advantages to explore novel superfluid phases, quantized vortices, collective modes, and many-body effects.

The collective oscillation frequencies and damping rates of cold Fermi gases can be precisely measured [6-10], which provides a good testing ground for different aspects of manybody theories. In particular, the breathing mode has been extensively studied [11], which is related to the equation of state and incompressibility. In the unitary limit, the Fermi gas is characterized by infinite scattering length and its dynamic properties are insensitive to interactions. The collective oscillation frequencies of large systems can be well described by the hydrodynamical approach $[12,13]$ and are insensitive to effective interactions. In contrast to large systems adopted in experiments, the detailed quantum effects such as shell effects and superfluidity can have large impacts for small systems. Small systems have a finite density of states and collective modes would be different from the continuous hydrodynamical approach. It is, therefore, crucial to establish a connection between small systems that can be described by microscopic approaches and large systems that can be described by the hydrodynamical approach.

\footnotetext{
*peij@pku.edu.cn
}

Some of the microscopic approaches, for example, the quantum Monte Carlo (QMC) method [14], are numerically very challenging for a large number of trapped particles. The suitable microscopic method to describe the ground state of Fermi gases is the Bogoliubov-de Gennes (BdG) equation. For unitary Fermi gases, the superfluid local density approximation (SLDA) [15] is an effective superfluid density functional theory (DFT) approach which was developed according to experiments and QMC simulations. The collective excitations can be in principle self-consistently described by the time-dependent $\mathrm{BdG}$ equation, or time-dependent density functional theory [16]. For small-amplitude collective motions, such as breathing vibrations, quasiparticle random phase approximation (QRPA) (or linear response theory) can match the real-time dynamical results and is more efficient. Several QRPA calculations have been performed for Fermi gases, and interesting insights have been achieved such as multipole collective modes [11,17], the Higgs modes [18], Goldstone modes [19,20], pair-breaking modes [20,21], and finite-size effects [22]. In addition, the transition currents in QRPA can directly reveal the dynamic mechanisms of collective modes [23]. In experiments, Fermi gases have usually been studied in highly elongated traps [5-10]. By varying the trap deformations in QRPA calculations to highly prolate (elongated) shapes, we can simulate the collective modes of quasi-one-dimensional systems. We can also explore the collective modes of quasi-two-dimensional (2D) systems with extremely oblate (pancake-shaped) deformed traps, which are also attractive in experiments and theories [24-27]. It is interesting to access the dimensional evolution by performing calculations in extremely deformed traps. However, previous QRPA calculations of Fermi gases are not really preformed within deformed traps. Actually, deformed QRPA has been widely applied in nuclear physics [28] but calculations are very time consuming. 
In this work, we aim to investigate the collective breathing modes of a unitary Fermi gas at zero temperature in very deformed traps, within the framework of QRPA. We can study the dynamical properties of systems in elongated and pancake-shaped traps with a small number of particles, e.g., 200 particles, due to large computational costs. The properties of such systems are also interesting for nuclear physics. To see the role of finite-size effects, we also studied large systems consisting of 2000 particles. The realistic particle numbers in experiments are usually about $10^{5}[6,7,9,10]$, which are too large for microscopic calculations. Recent experiments on radio-frequency spectroscopy performed in quasi-2D systems employed about 1000 atoms [24,25]. Such a finitesize system is well suited for our approach. The calculations are performed in axial-symmetric discretized coordinate spaces based on $B$ splines [29]. The ground states of unitary Fermi gases are described by SLDA with different parameters [15]. The QRPA equation is based on wavefunctions from SLDA and is solved by an iterative method, known as the finite-amplitude method [30,31], instead of standard diagonalization of a huge matrix [28]. The used iterative solving method is much more efficient and has been widely applied in finite quantum systems such as nuclear and chemical physics. The results include transition strengths as a function of oscillation frequencies, and also transition currents which can reveal the dynamic mechanisms of collective modes.

\section{THEORETICAL FRAMEWORK}

\section{A. Superfluid local density approximation}

For the unitary Fermi gas, the system exhibits a universal behavior governed by the densities, making it ideally suited for a DFT description [15,32]. DFT can incorporate experimental information and quantum Monte Carlo results within an accurate energy density functional and is able to describe strongly interacting systems due to the incorporation of many-body effects. In this respect, SLDA, a superfluid extension of DFT, was developed by Bulgac and it has been applied to symmetric two-component systems [15]. Timedependent SLDA calculations [16] and SLDA random phase approximation (RPA) calculations [19] have been demonstrated to be very useful for studies of excitation properties of Fermi gases. The SLDA has been generalized to asymmetric superfluid local density approximation (ASLDA) for spinimbalanced systems [33], which predicts the existence of Larkin-Ovchinnikov phases in elongated traps [34].

We consider that the symmetric unitary Fermi gas is trapped by an axial-symmetric external potential, $V_{\text {ext }}(\boldsymbol{r})=$ $\frac{1}{2} m \omega_{T}^{2}\left(r^{2}+z^{2} / \zeta^{2}\right)$, where $\zeta$ denotes the elongation of the trap and the $z$ axis is the principle axis. We work in natural trap units for which $\hbar=\mathrm{m}=\omega_{T}=1$. The SLDA energy density functional is written as [15]

$$
\begin{aligned}
\epsilon(\boldsymbol{r})= & \alpha \frac{\tau(\boldsymbol{r})}{2}+\beta \frac{3\left(3 \pi^{2}\right)^{\frac{2}{3}} \rho^{\frac{5}{3}}(\boldsymbol{r})}{10}-\Delta(\boldsymbol{r}) \kappa(\boldsymbol{r}) \\
& +V_{\mathrm{ext}}(\boldsymbol{r}) \rho(\boldsymbol{r})
\end{aligned}
$$

where the particle densities $\rho(\boldsymbol{r})$, kinetic energy density $\tau(\boldsymbol{r})$, pairing density $\kappa(\boldsymbol{r})$, and pairing potential $\Delta(\boldsymbol{r})$ are written as

$$
\begin{aligned}
& \rho(\boldsymbol{r})=2 \sum_{E_{k}<E_{c}}\left|v_{k}(\boldsymbol{r})\right|^{2}, \quad \tau(\boldsymbol{r})=2 \sum_{E_{k}<E_{c}}\left|\nabla v_{k}(\boldsymbol{r})\right|^{2}, \\
& \kappa(\boldsymbol{r})=\sum_{E_{k}<E_{c}} v_{k}^{*}(\boldsymbol{r}) u_{k}(\boldsymbol{r}), \quad \Delta(\boldsymbol{r})=-g_{\mathrm{eff}}(\boldsymbol{r}) \kappa(\boldsymbol{r}) .
\end{aligned}
$$

The effective pairing strength $g_{\text {eff }}(\boldsymbol{r})$ is obtained by renormalization according to an ultraviolet cutoff $\Lambda_{c}$ [15]:

$$
\frac{1}{g_{\mathrm{eff}}(\boldsymbol{r})}=\frac{\rho^{\frac{1}{3}}(\boldsymbol{r})}{\gamma}+\Lambda_{c}(\boldsymbol{r})
$$

In the above equations, the SLDA parameters $\alpha, \beta$, and $\gamma$ are dimensionless constants.

Minimization of the energy density functional leads to a BdG set of equations:

$$
\left(\begin{array}{cc}
h(\boldsymbol{r})-\mu & \Delta(\boldsymbol{r}) \\
\Delta^{*}(\boldsymbol{r}) & -\left(h^{*}(\boldsymbol{r})-\mu\right)
\end{array}\right)\left(\begin{array}{c}
u_{k}(\boldsymbol{r}) \\
v_{k}(\boldsymbol{r})
\end{array}\right)=E_{k}\left(\begin{array}{c}
u_{k}(\boldsymbol{r}) \\
v_{k}(\boldsymbol{r})
\end{array}\right),
$$

where the single-particle Hamiltonian $h$ is determined by variation of the SLDA energy density functional with respect to $\tau$ and $\rho$ densities. The densities are constructed from quasiparticle wave functions $u_{k}(\boldsymbol{r})$ and $v_{k}(\boldsymbol{r})$.

Note that the SLDA form in Eq. (1) breaks the Galilean invariance, and therefore the contribution of the current density $\vec{j}(\boldsymbol{r})$ should be included:

$$
\begin{aligned}
\epsilon(\boldsymbol{r})= & \alpha \frac{\tau_{c}(\boldsymbol{r})}{2}+\beta \frac{3\left(3 \pi^{2}\right)^{\frac{2}{3}} \rho^{\frac{5}{3}}(\boldsymbol{r})}{10}+g_{\mathrm{eff}}\left|\kappa_{c}(\boldsymbol{r})\right|^{2} \\
& +V_{\mathrm{ext}}(\boldsymbol{r}) \rho(\boldsymbol{r})-(\alpha-1) \frac{\vec{j}^{2}(\boldsymbol{r})}{2 \rho(\boldsymbol{r})},
\end{aligned}
$$

where the current density $\vec{j}(\boldsymbol{r})$ is written as

$$
\vec{j}(\boldsymbol{r})=2 \operatorname{Im} \sum_{E_{k}<E_{c}} v_{k}^{*}(\boldsymbol{r}) \nabla v_{k}(\boldsymbol{r}) .
$$

The current density has no contribution to ground states of spin-balanced systems, but it plays a role in excited states.

The parameters $\alpha, \beta$, and $\gamma$ in the SLDA energy density functional can be related to physical parameters $\alpha, \xi$, and $\eta$ [15]. The parameter $\alpha$ is defined by the effective mass, $\alpha=$ $m / m_{\text {eff }}$. The parameters $\xi$ and $\eta$ are defined by the particlenumber density $\rho=N / V=\frac{k_{F}^{3}}{3 \pi^{2}}$, the chemical potential $\mu=$ $\xi \epsilon_{F}$ ( $\xi$ is called the Bertsch parameter), and the pairing gap $\Delta=\eta \epsilon_{F}$ of the homogeneous Fermi gas. These physical quantities can be determined by QMC calculations [14,35] and experimental measurements [36]. The relation between SLDA parameters $\beta$ and $\gamma$ and physical parameters $\xi$ and $\eta$ are given by coupled integral equations [15,37]. In the latest experiment performed in the unitary, the obtained Bertsch parameter $\xi$ in three-dimensional (3D) systems is 0.376 [38]. The pairing gap parameter $\eta$ at unitary has been measured to be about 0.44 [39] and more experiments are needed. Note that these relation parameters could be dependent on 
TABLE I. The five sets of SLDA parameters adopted in this work.

\begin{tabular}{lccccc}
\hline \hline Parameter sets & $\mathrm{a}$ [41] & $\mathrm{b}$ [14] & $\mathrm{c} \mathrm{[37]}$ & $\mathrm{d}$ [19] & $\mathrm{e}$ [42] \\
\hline$\alpha$ & 1.14 & 1.12 & 1.104 & 1.00 & 0.812 \\
$\beta$ & -0.553 & -0.520 & -0.417 & -0.430 & -0.712 \\
$1 / \gamma$ & -0.0906 & -0.0955 & -0.0347 & -0.0767 & -0.0705 \\
$\xi$ & 0.422 & 0.440 & 0.374 & 0.376 & 0.449 \\
$\eta$ & 0.504 & 0.486 & 0.651 & 0.500 & 0.442 \\
\hline \hline
\end{tabular}

the finite-size effects [40]. There are several sets of parameters $\alpha, \beta$, and $\gamma$ adopted in the literature, as shown in Table I.

\section{B. Finite-amplitude method for quasiparticle random phase approximation}

The standard QRPA equation includes many particle-hole excitations [28], and the resulting configuration space is huge for deformed cases and continuum. For example, with 1000 single-particle levels, the matrix which needs to be diagonalized would have a dimension of the order of $\sim 10^{6} \times 10^{6}$. On the other hand, the QRPA equation can be derived from the time-dependent Hartree-Fock-Bogoliubov equation by assuming that the collective motion has small amplitudes [30]. Here we refer to the finite-amplitude method for QRPA (FAM-QRPA), which allows to solve QRPA iteratively and much more efficiently, by avoiding the calculation of a huge configuration matrix. The FAM-QRPA has been applied to multipole giant resonances and pygmy resonances in nuclei $[23,30,31,43]$. Our solver is implemented in the cylindrical coordinate space which is useful for descriptions of very deformed and weakly bound systems. Note that the collective modes are temperature dependent [44] and currently we only study the zero-temperature unitary Fermi gases.

In FAM-QRPA, different collective modes of systems are obtained by responses to various external fields with oscillating frequencies $\omega$. We solve the nonlinear FAM-QRPA equations as follows [30]:

$$
\begin{aligned}
& \left(E_{\mu}+E_{\nu}-\omega\right) X_{\mu \nu}(\omega)+\delta H_{\mu \nu}^{20}(\omega)=-F_{\mu \nu}^{20}(\omega), \\
& \left(E_{\mu}+E_{\nu}+\omega\right) Y_{\mu \nu}(\omega)+\delta H_{\mu \nu}^{02}(\omega)=-F_{\mu \nu}^{02}(\omega),
\end{aligned}
$$

where $\delta H_{\mu \nu}^{20}(\omega)$ and $\delta H_{\mu \nu}^{02}(\omega)$ are the induced Hamiltonian. $F_{\mu \nu}^{20}$ and $F_{\mu \nu}^{02}$ correspond to the external field, for which $F=$ $r^{L} Y_{L K}(\theta, \varphi)$ is the $L$ th-order operator for multipole modes. For the monopole mode (breathing mode), $F$ takes $F=$ $r^{2} Y_{00}(\theta, \varphi)$. We solve the transition amplitudes $X(\omega)$ and $Y(\omega)$ iteratively at each collective excitation frequency $\omega$. To smooth the transition strength, an artificial imaginary part is included in the frequency as $\omega+i \Gamma$. Thus the resulting resonance widths of collective modes indicate relative damping widths rather than real damping widths. The nonlinear FAMQRPA equation is solved by using the modified Broyden method [45].

The induced fields can be obtained by linear functions of induced densities. For SLDA, the induced mean-field potential $\delta U$, induced pairing potential $\delta \Delta$, and induced timeodd field $\delta h_{\text {odd }}$ can be written as

$$
\begin{gathered}
\delta U=\beta \frac{\left(3 \pi^{2}\right)^{\frac{2}{3}}}{3 \rho^{\frac{1}{3}}} \delta \rho-\left(\frac{g_{\text {eff }}}{3 \gamma \rho^{\frac{2}{3}}}\right)^{2}\left(\kappa^{*} \Delta+\Delta^{*} \kappa\right) \delta \rho \\
+\frac{g_{\text {eff }}}{3 \gamma \rho^{\frac{2}{3}}}\left(\Delta \delta \kappa^{-}+\Delta^{*} \delta \kappa^{+}\right)+\frac{2|\Delta|^{2}}{9 \gamma \rho^{\frac{5}{3}}} \delta \rho, \\
\delta \Delta^{ \pm}=\frac{g_{\text {eff }} \Delta}{3 \gamma \rho^{\frac{2}{3}}} \delta \rho-g_{\text {eff }} \delta \kappa^{ \pm}, \\
\delta h_{\text {odd }}=\frac{i}{2 \rho}(\alpha-1)(\nabla \cdot \delta \vec{j}+\delta \vec{j} \cdot \nabla) .
\end{gathered}
$$

The induced fields are based on transition density $\delta \rho$, transition pairing density $\delta \kappa$, and transition current $\delta \vec{j}$. These transition densities are constructed from the set of quasiparticle wavefunctions $U$ and $V$ and transition amplitudes $X$ and $Y$. The various transition densities are given as

$$
\begin{gathered}
\delta \rho=U X V^{T}+V^{*} Y^{T} U^{\dagger}, \\
\delta \kappa^{+}=U X U^{T}+V^{*} Y^{T} V^{\dagger}, \\
\delta \kappa^{-}=V^{*} X^{\dagger} V^{\dagger}+U Y^{*} U^{T}, \\
\delta \vec{j}=\frac{1}{2 i}\left[\left(\nabla U^{*}\right) Y V^{*}+U X(\nabla V)\right. \\
\left.-(\nabla U) X V-U^{*} Y\left(\nabla V^{*}\right)\right] .
\end{gathered}
$$

Finally the total transition strength function $S(F ; \omega)$ of collective modes can be obtained from the converged transition amplitudes $(X, Y)$ of FAM-QRPA solutions as

$$
S(F ; \omega)=-\frac{1}{\pi} \operatorname{Im} \sum_{\mu<\nu}\left[F_{\mu \nu}^{20 *} X_{\mu \nu}(\omega)+F_{\mu \nu}^{02 *} Y_{\mu \nu}(\omega)\right] \text {. }
$$

\section{RESULTS AND DISCUSSIONS}

\section{A. Unitary Fermi gases in spherical traps}

We first consider a unitary Fermi gas of 200 particles within a spherical harmonic trap. The numerical accuracy depends on the sizes of coordinate spaces and mesh spacing. The unit of the length scale should be $\sqrt{\hbar /\left(m \omega_{T}\right)}$, which is 1 in the natural unit in this work. The mesh spacing is taken as 0.2 in this work. The adopted energy cutoff is about 20 for renormalization. First, the ground states of the systems in a spherical trap are calculated. The ground-state properties are relevant for collective oscillation frequencies. Figure 1 shows the number density and pairing potential with different SLDA parameters as shown in Table I. We see the densities are more or less similar for different parameters. However, parameter set (c) resulted in a particularly large pairing gap which has the largest pairing strength of $1 / \gamma=-0.0347$.

The calculated monopole excitation peak frequencies $\omega$ with different SLDA parameters are give in Table II. The unit of the excitation frequency in this work is in terms of the trap frequency, i.e., $\omega / \omega_{T}$. The peak frequency can also be estimated by $\omega=m_{1} / m_{0}$, where the energy-weighted sum rule is defined as $m_{k}=\int d \omega S(F ; \omega) \omega^{k}$ [46]. We see larger discrepancies in peak- $\omega$ values for the different parameter sets used. The largest frequency $\omega$ is obtained with parameter 


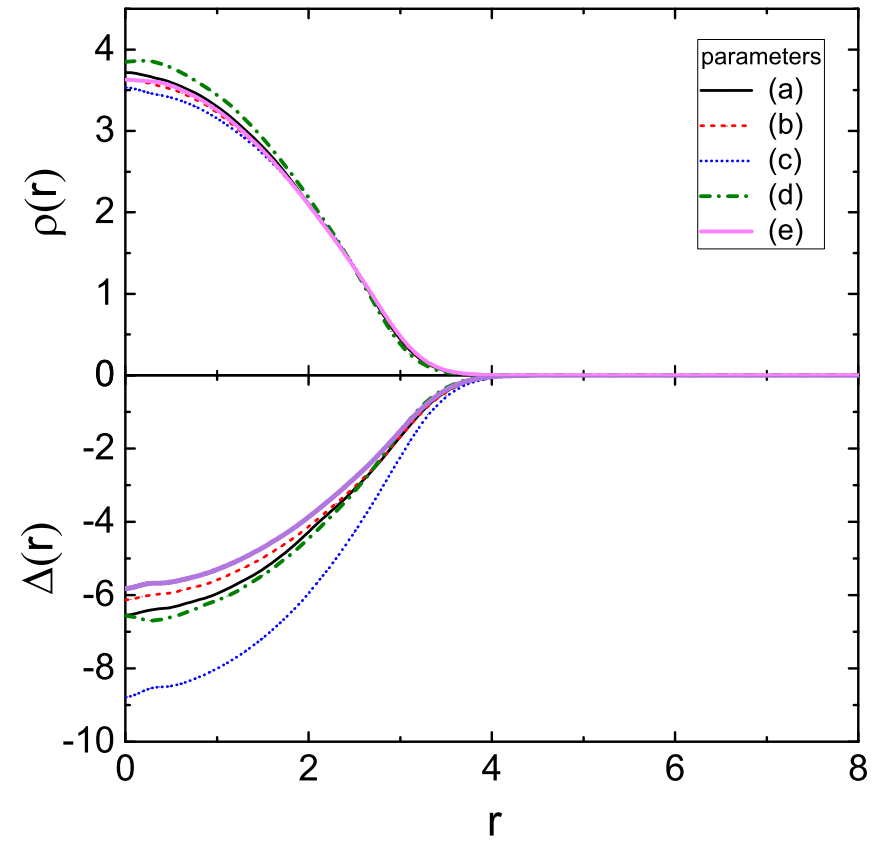

FIG. 1. The ground-state particle densities $\rho(r)$ and pairing gaps $\Delta(r)$ with 200 particles in a spherical trap. Calculations are performed with different SLDA parameters as given in Table I. The unit of length coordinate $r$ is $\sqrt{\hbar /\left(m \omega_{T}\right)}$, which is 1 in natural units.

(c) in Table I, which corresponds to the largest pairing strength. In Table II, the differences between $m_{1} / m_{0}$ and $\omega$ from peaks are rather small. The lowest $\omega$ is obtained from parameter (e), corresponding to the smallest $\alpha=0.812$ and the largest effective mass. In analogy to nuclear monopole resonances, the breathing mode peak frequencies are inversely proportional to $\sqrt{m^{*}}$ [47]. In addition, the monopole frequencies are also inversely proportional to the rms radii [46]. Small systems have small radii and thus the frequencies are very large. The obtained $\omega$ values of systems of 200 particles are much larger than the hydrodynamical result of $\omega=2$ in spherical traps [12,13]. For large systems, it is known that QRPA calculations of $10^{4}$ particles in a spherical trap can match hydrodynamical results [22]. For small systems, we see that quantum effects play a significant role and it is a good testing ground for different parameters.

To further analyze the role of different SLDA parameters, we display different contributions to transition strengths related to, e.g., the mean field, the pairing field, and the time-

TABLE II. The monopole oscillation peak frequencies of 200 particles in a spherical trap, calculated with SLDA-QRPA with different parameters. The peak frequencies are compared with the sum rule results $m_{1} / m_{0}$. The ground-state energies and rms radii are also given.

\begin{tabular}{lccccc}
\hline \hline Systems & $\mathrm{a}$ & $\mathrm{b}$ & $\mathrm{c}$ & $\mathrm{d}$ & $\mathrm{e}$ \\
\hline$\omega$ (FAM-QRPA) & 6.261 & 6.033 & 6.943 & 5.951 & 4.738 \\
$m_{1} / m_{0}$ & 6.250 & 6.054 & 7.021 & 6.008 & 4.804 \\
$E_{g s}$ & 840.6 & 859.8 & 795.4 & 795.0 & 857.5 \\
$\sqrt{\left\langle r^{2}\right\rangle}$ & 2.15 & 2.17 & 2.17 & 2.11 & 2.16 \\
\hline \hline
\end{tabular}

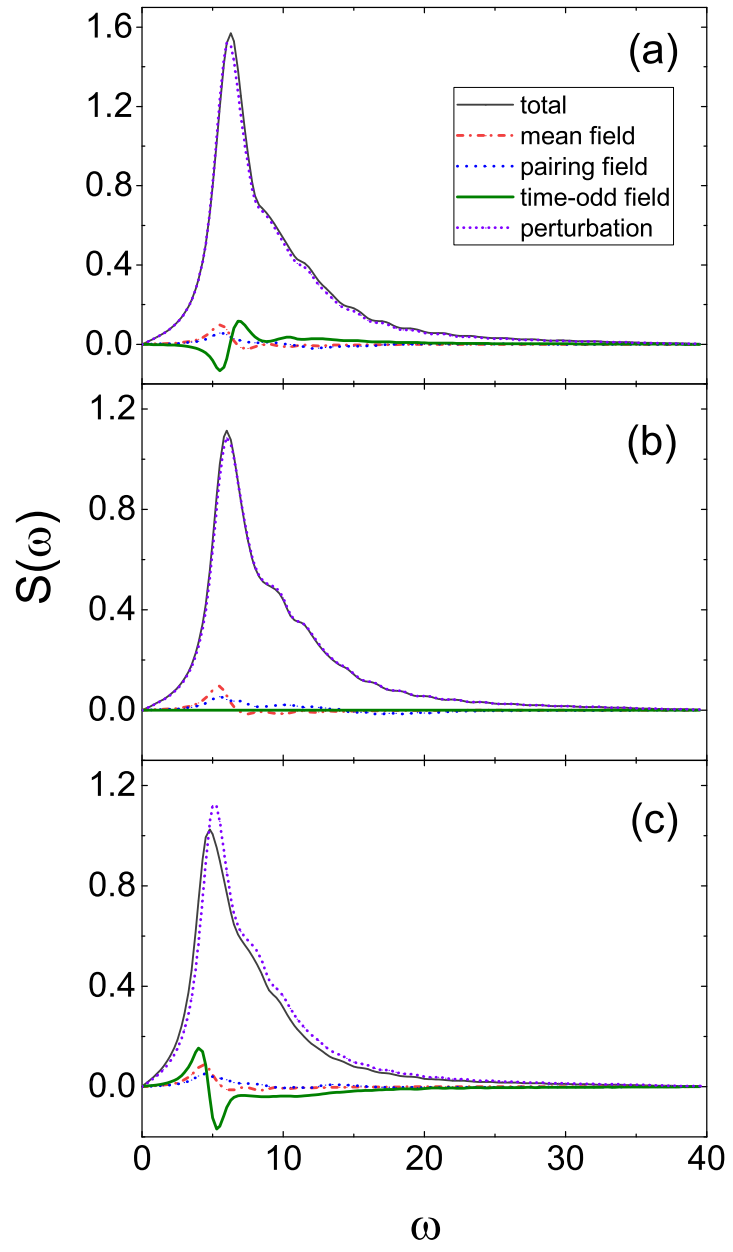

FIG. 2. The total response strengths and contributions of induced fields given by SLDA-QRPA with 200 particles in a spherical trap with different SLDA parameter sets (a), (d), and (e), respectively, as listed in Table I. The unit of the frequency $\omega$ is in terms of $\omega / \omega_{T}$ and is dimensionless.

odd field, as shown in Fig. 2. In Fig. 2, transition strengths corresponding to the three parameter sets (a), (d), and (e) are shown. Note that the calculated transition strengths are given with an artificial smoothing parameter $\Gamma$ of 0.2 , which is smaller than the trap frequency. The main contribution to the total response strength is from the external perturbation field, which is determined by ground-state properties. The contribution of induced mean field has a lower frequency than the main peak frequency. The contribution of induced pairing fields is not significant. The induced time-odd field, based on the transition current $\delta \vec{j}$, plays a significant role when the effective mass is not equal to 1 . For $\alpha$ smaller than 1 in parameter set (e), the $\delta \vec{j}$ field has a positive contribution in the low-energy regime and thus shifts the frequency remarkably to low energies (see Table II). In contrast, for $\alpha$ larger than 1 in parameter set (a), the $\delta \vec{j}$ field has an oppositive contribution and shifts the frequency to high energies. This is consistent with the role of effective mass, in which a large effective mass (or a small $\alpha$ ) results in a small monopole frequency. We demonstrated that the induced time-odd term and the effective 


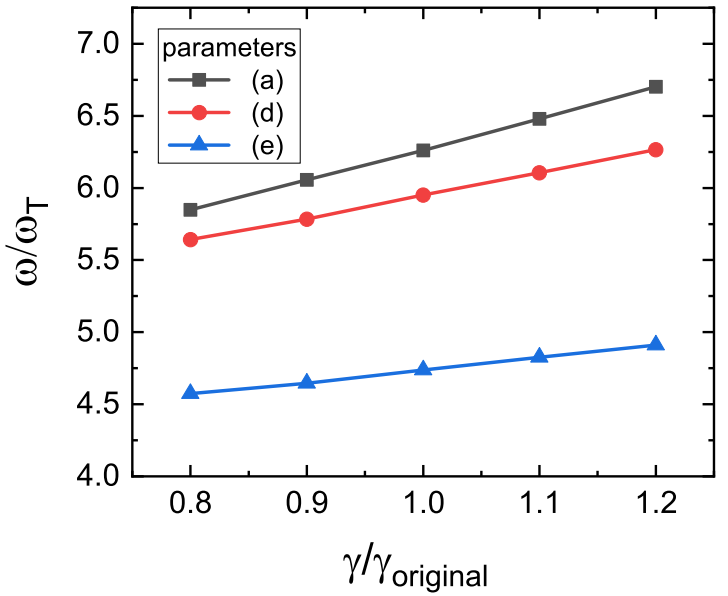

FIG. 3. The monopole oscillation frequencies of 200 particles in a spherical trap with varying pairing parameters $1 / \gamma$. Calculations are performed with SLDA parameter sets (a), (d), and (e), as listed in Table I.

mass have significant consequences in collective modes of small systems.

The role of pairing in the equation of state and collective modes is very interesting. For example, it is interesting to know the differences between superfluid and normal hydrodynamics [48]. The monopole frequencies with varying pairing interaction strengths are given in Fig. 3. Note that the influences of pairing include the static pairing and included pairing. We see that with parameter set (a), the monopole frequency increased linearly by $7 \%$ when pairing strength increased by $20 \%$. The dependence on pairing strength is less prominent for cases with smaller excitation frequencies. This explains that parameter set (c) with the largest pairing strength [about 2.6 times larger than (a)] corresponds to the largest monopole frequency, compared to other parameter sets. The monopole frequencies $\omega$ are less affected even if we turn off the induced pairing interactions, implying the static pairing rather than the induced pairing is dominant. Indeed, the largest monopole peak frequency $\omega$ is related to the largest groundstate pairing field $\Delta$ at the trap center as shown in Fig. 1. In the cold Fermi gases that are close to unitary, the pairing plays a role to increase the collective oscillation frequency [22]. In contrast, the pairing can increase collectivity and shifts down nuclear giant monopole resonance energies [46,49]. On the other hand, the pairing can increase frequencies of nuclear pygmy resonances associated with dilute halo surfaces [50], which is similar to unitary Fermi gases. It is understandable that the stronger pairing can increase quasiparticle energies and shift up the oscillation frequencies. Note that calculations in this work are performed at zero temperature, and a finite temperature in experiments can significantly reduce the pairing. We see that the monopole frequency of a small spherical system can be very much dependent on SLDA parameters. We return to this observation in the later part.

\section{B. Collective frequencies of deformed systems}

We have studied monopole modes of a unitary Fermi gas of 200 particles in prolate (elongated shape) traps,

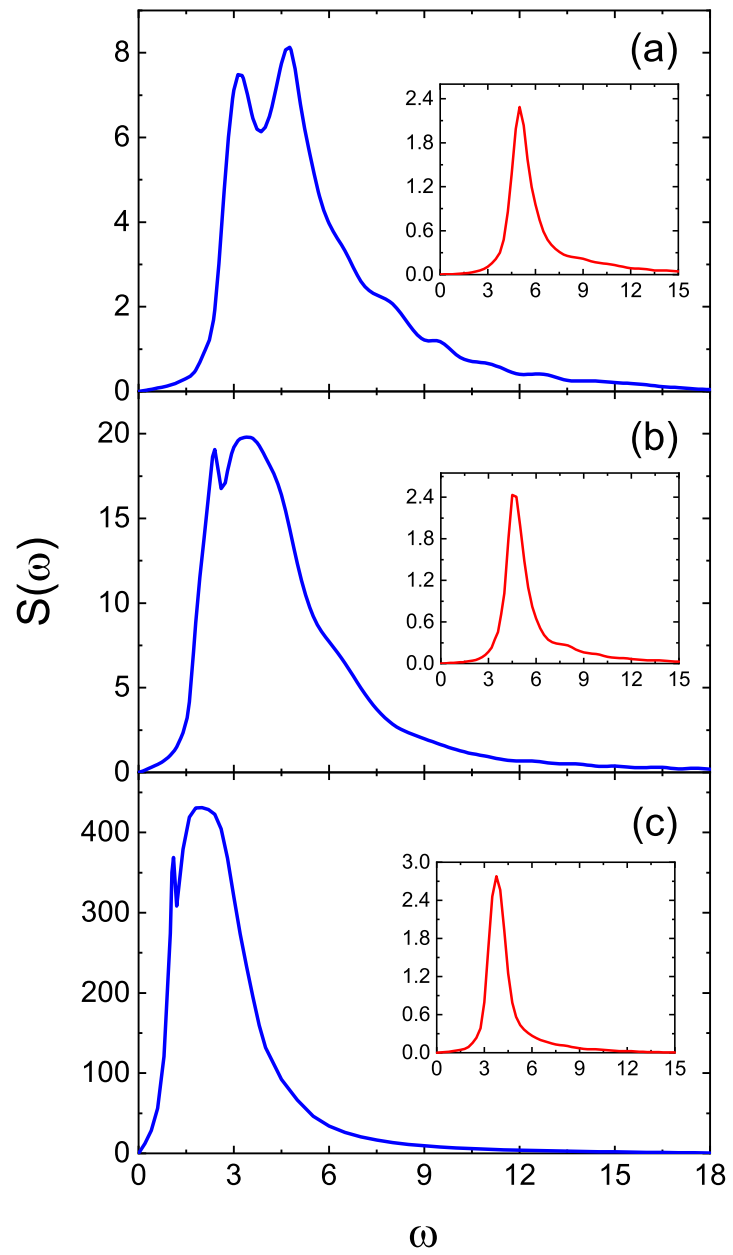

FIG. 4. The transition strengths of monopole mode of 200 particles in prolate traps with trap aspect ratios of (a) $\zeta=3$, (b) $\zeta=$ 5 , and (c) $\zeta=20$. The insets show the corresponding transition strengths of radial modes.

$V_{\text {ext }}(\boldsymbol{r})=\frac{1}{2} m \omega_{T}^{2}\left(r^{2}+z^{2} / \zeta^{2}\right)$, and oblate (pancake-shaped) traps, $V_{\text {ext }}(\boldsymbol{r})=\frac{1}{2} m \omega_{T}^{2}\left(r^{2} / \zeta^{2}+z^{2}\right)$. The trap aspect ratio is defined by $\zeta$. The collective transition strengths of prolate and oblate traps with parameters set (a) are shown in Figs. 4 and Fig. 5, respectively.

In Fig. 4 for elongated traps, the monopole transition strength with $\zeta=3$ has two peaks at frequencies of 3.21 and 4.71 , respectively. With $\zeta=5$, the first peak is shifted to a lower frequency of 2.42 and the second peak becomes prominent with an energy of 3.47 . With increasing $\zeta$, the second peak becomes dominant and the first peak becomes narrower. With $\zeta=20$, the frequencies of two axial modes are 1.1 and 2.2, and the radial mode is at 3.75. The calculated radial modes are also displayed as insets in Fig. 4. It is known that the axial modes and radial modes are separated in well-deformed cases [23]. As shown, the radial modes have a single peak which slowly shifts to low energies as deformation increases. The collective resonances are obtained with the same smoothing parameter $\Gamma$. Thus the widths of collective modes are useful for studying the relative damping rates. The widths of the first axial mode and the radial mode become narrower with increasing deformations. In elongated 


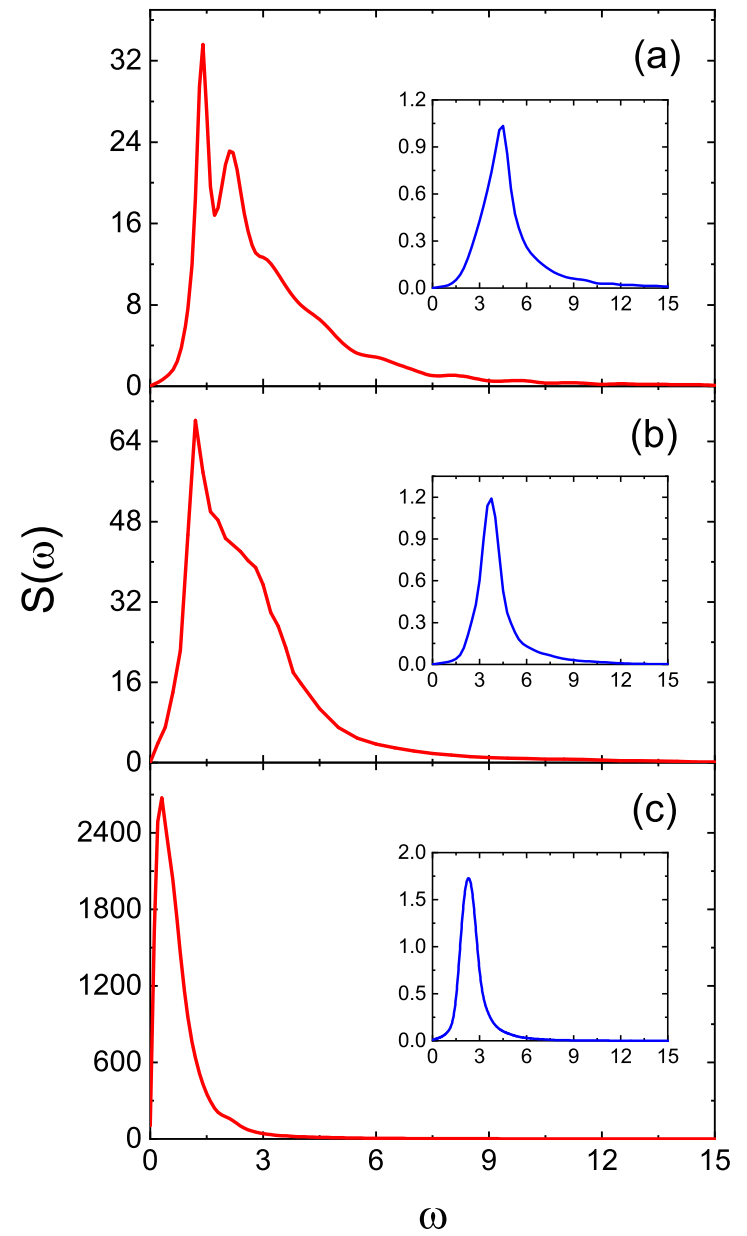

FIG. 5. The transition strengths of monopole mode of 200 particles in oblate traps with trap aspect ratios of (a) $\zeta=3$, (b) $\zeta=5$, and (c) $\zeta=20$. The insets show the corresponding transition strengths of axial modes.

traps, however, the transition strengths of radial modes are too small compared to axial modes. The appearance of the second mode in elongated traps is rather unusual and we discuss it in following sections.

In oblate traps as shown in Fig. 5, the modes correspond to a small deformation $\zeta=3$ also have two peaks. With increasing $\zeta$, the main peak shifts to lower frequency and becomes narrower. At $\zeta=20$, the second peak disappears and the breathing mode is a single narrow resonance. With $\zeta=20$, the frequency of the radial mode is 0.3 , and the axial mode is at 2.4. The axial modes are suppressed in pancakeshaped traps. The axial modes also shift to lower energies and become narrower, approaching the hydrodynamical limit. The widths of radial and axial modes in pancake-shaped traps are comparable.

In both elongated and pancake-shaped traps, the obtained peak frequencies are much higher than the quasi-1D $(\omega=$ $\left.\sqrt{12 / 5} \omega_{z}\right)$ and quasi-2D $\left(\omega=\sqrt{3} \omega_{r}\right)$ hydrodynamical results [13]. The deviation is particularly significant in elongated traps. The peak frequencies actually decrease slowly with increasing prolate deformations, while the deformation scaling is more reasonable in oblate traps. In contrast, the radial modes in elongated traps and axial modes in pancake-
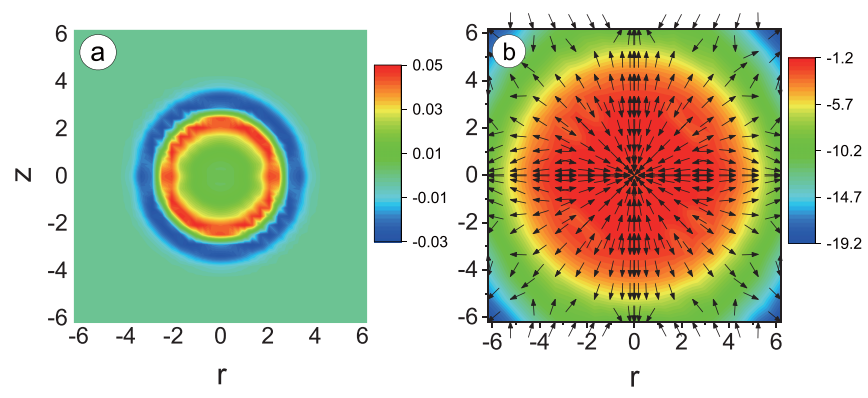

FIG. 6. (a) Calculated induced transition density $\delta \rho(r, z)$ and (b) induced current density $\delta \vec{j}(r, z)$ of monopole modes of a spherical system of 200 particles. Results are displayed in cylindrical coordinates $(r, z)$.

shaped traps are approaching the hydrodynamical results of quasi-1D $\left(\omega=\sqrt{10 / 3} \omega_{r}\right)$ and quasi-2D $\left(\omega=\sqrt{8 / 3} \omega_{z}\right)$ systems [13] as trap deformation increases. The differences in collective frequencies due to different parameters also decreased at large deformations.

\section{Transition densities and currents}

In QRPA calculations, the obtained transition densities $\delta \rho$ and transition currents $\delta \vec{j}$ can directly reveal the dynamical mechanism of collective modes. An additional interesting ingredient is provided by the superfluidity and its effects on the flow pattern. The transition densities and currents are also major ingredients in the hydrodynamical approach. It is possible that the transition densities and currents can be obtained by analysis of angle-resolved scattering experiments. It is known that the flow patterns of collective modes are dependent on excitation energies and deformations [23].

To study the mechanisms of collective modes in different traps, we calculated the transition densities and currents with the SLDA parameter of $\alpha=1.14, \beta=-0.553$, and $1 / \gamma=$ -0.0906 . The results of the spherical trap are shown in Fig. 6 . For the transition density, a ring structure appears with a radius of 3 , indicating that the breathing mode is a surface mode. The transition density illustrates clearly the breathing behavior with oppositive values between inside and outside rings. The integral of the total transition density is close to zero. For the transition current, the compressional flow is inward within the ring, but the flow is outward beyond the ring. This is because the velocity changes direction at the outer surface, where the density is about $12 \%$ of the center density. Note that the same time-evolution forms of oscillating transition densities and currents are assumed. We illustrated that the compressional flow in spherical cases has a simple pattern.

For deformed systems, we show the transition densities and currents in the elongated traps with $\zeta=5$ in Fig. 7. There are two resonance peaks at $\omega=2.42$ and $\omega=3.47$. From the transition densities, we can see that both modes are mainly related to vibrations at axial ends of the elongated system, indicating both modes are axial modes. From the transition currents, we can see the lowest mode has a clear compressional flow pattern towards the center. The flow pattern has three nodes in the axial direction. For the second peak, the 

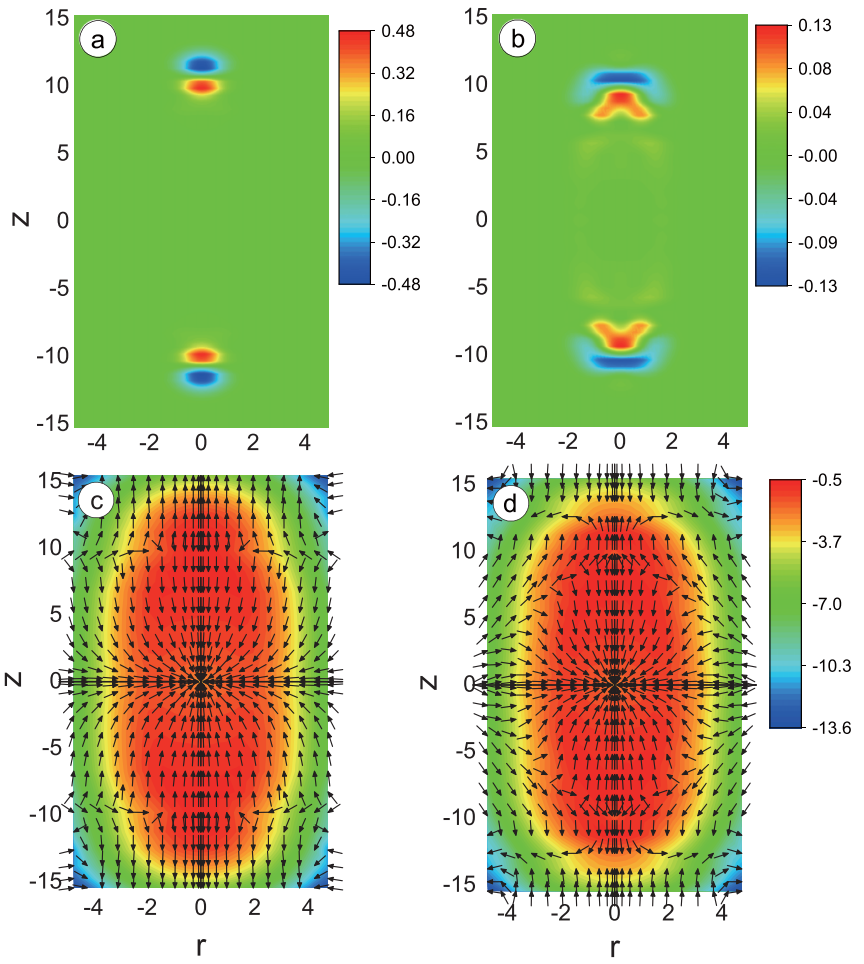

FIG. 7. (a) For systems with 200 particles within an elongated trap of $\zeta=5$, calculated induced transition density $\delta \rho(r, z)$ : (a) for peak at 2.42 and (b) for peak at 3.47. Calculated induced current density $\delta \vec{j}(r, z)$ : (c) for peak at 2.42 and (d) for peak at 3.47.

transition current is more complex and its transition density involves a larger spatial region, associated with a higher excitation frequency. Actually, the current of the second peak has five nodes in the axial direction, which is distinct from the first peak with three nodes. Naturally the current patterns become complex as excitation energies increase, in analogy to quantum topology excitations.

The transition density and currents of the oblate $\zeta=5$ trap are shown in Fig. 8. The transition density shows that the vibrations are mainly at the surrounding edge of the pancake-shaped system. The transition currents indicate that it is a simple radial mode. Close to the center, the flow is inwards and the current has three nodes in the radial axis. Actually the flow of the pancake-shaped system is similar to the flow of the first peak in the elongated system when rotated by $90^{\circ}$. The transition densities show that collective modes in elongated and pancake-shaped systems are axial and radial modes, respectively. However, the transition currents show that the collective modes are nontrivial as the presence of several nodes indicates. A full 3D calculation should be adopted for more realistic studies of flow patterns.

\section{Towards large systems}

It is interesting to study the evolution of collective modes from small to large systems. However, it is a very demanding task for SLDA and QRPA calculations of large systems in deformed traps. We performed calculations of 2000 particles in deformed traps, to compare with systems of 200 particles. It has been shown that QRPA calculations of $10^{4}$ particles

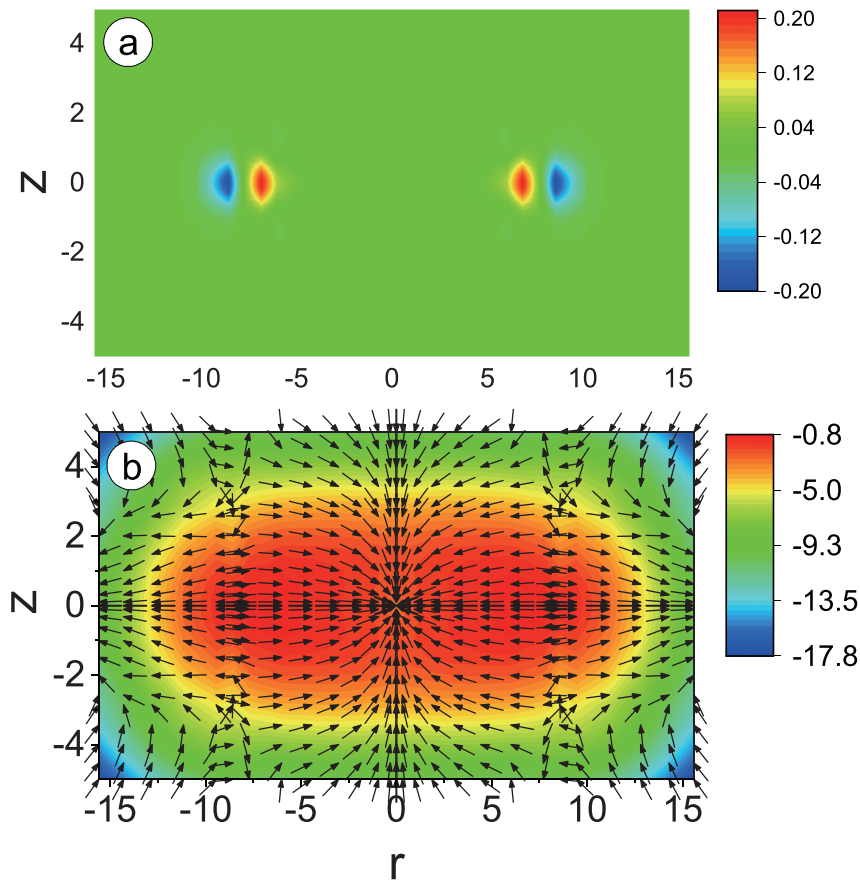

FIG. 8. (a) Calculated induced transition density and (b) induced current density of a system of 200 particles in a pancake-shaped trap with $\zeta=5$. The corresponding frequency of the monopole mode is 1.24 .

can match the hydrodynamical approach [22]. In Ref. [22], calculations are performed based on the mean-field approximation in spherical traps at the BCS side of the crossover. However, with the parameters in Table I, our SLDA-QRPA calculations of 2000 particles in the spherical trap give an even higher excitation frequency than that of 200 particles. This is obviously inconsistent with the finite-size scaling and is mainly because the SLDA pairing strength is too large compared to Ref. [22]. We see that the finite-size scaling of collective frequencies breaks down due to a very large pairing strength.

To simulate the mean-field approach, in which the pairing strength $g=4 \pi \hbar^{2} a_{s} / m$ [22] and $a_{s}$ is the $s$-wave scattering length, we adopt a SLDA parameter set as $\alpha=1, \beta=$ -0.509 , and $1 / \gamma=-0.41$, corresponding to strong-coupling systems with $k_{F} a_{s}=-0.6$. In this case, the pairing strength is significantly decreased compared to the parameter sets in Table I, while other parameters have not been changed much. Actually, the finite-size dependence is significantly stronger in the pairing gap than in the Bertsch parameter $\xi$ [40].

In Fig. 9, the calculated monopole modes of 200 particles with the reduced pairing are shown. Figure 9 (a) shows the transition strength in a spherical trap with $1 / \gamma=-0.401$. The monopole peak frequency is 2.78 , which is much lower than the results shown in Table II. We demonstrated again that the monopole mode is shifted to lower frequency with reduced pairing. Figure 9(b) shows similar calculations in an elongated trap with $\zeta=20$. The monopole mode is now a single peak with the peak frequency of 0.53 . Last, Fig. 9(c) shows similar calculations in an elongated trap with $\zeta=20$ but with $1 / \gamma=-0.2$. In this case, the monopole mode has 


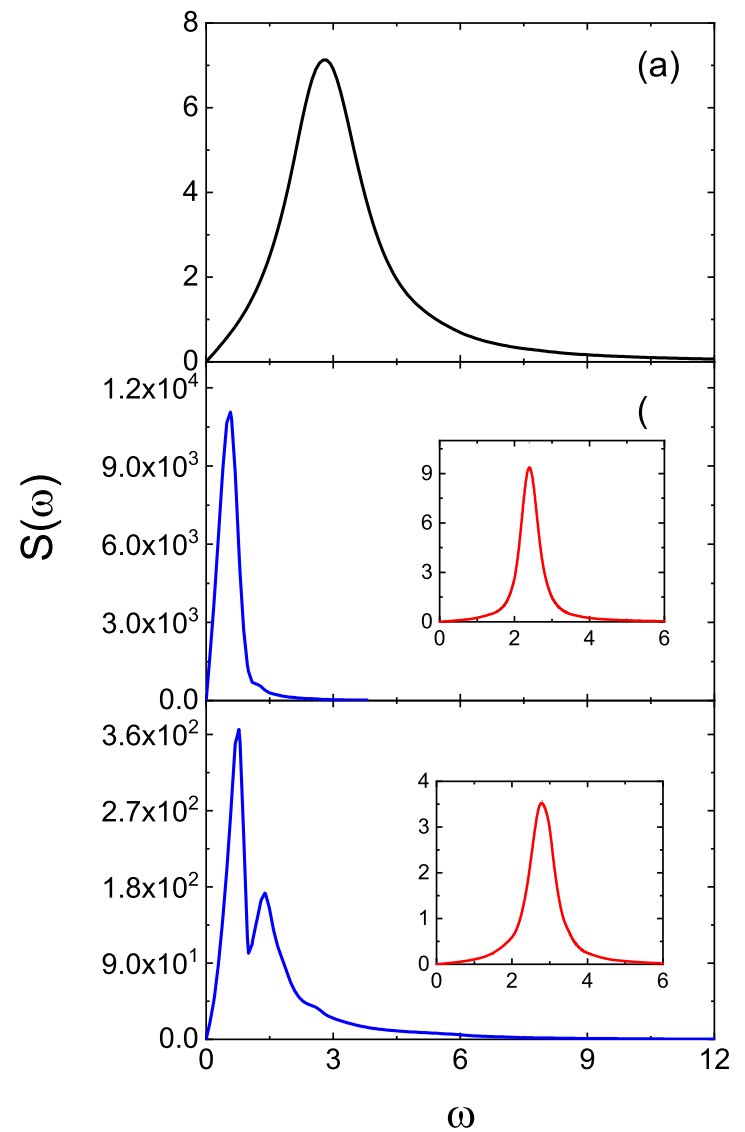



[24] M. Feld, B. Frohlich, E. Vogt, M. Koschorreck, $\begin{array}{llll}\text { and M. Kohl, Nature (London) 480, } 75 & \end{array}$ (2011).

[25] Y. Zhang, W. Ong, I. Arakelyan, and J. E. Thomas, Phys. Rev. Lett. 108, 235302 (2012).

[26] J. Hofmann, Phys. Rev. Lett. 108, 185303 (2012).

[27] B. C. Mulkerin, X. J. Liu, and H. Hu, Phys. Rev. A 97, 053612 (2018).

[28] P. Ring and P. Schuck, The Nuclear Many-Body Problem (Springer, Berlin, 1980).

[29] J. C. Pei, M. V. Stoitsov, G. I. Fann, W. Nazarewicz, N. Schunck, and F. R. Xu, Phys. Rev. C 78, 064306 (2008).

[30] P. Avogadro and T. Nakatsukasa, Phys. Rev. C 84, 014314 (2011).

[31] T. Nakatsukasa, T. Inakura, and K. Yabana, Phys. Rev. C 76, 024318 (2007).

[32] T. Papenbrock, Phys. Rev. A 72, 041603(R) (2005).

[33] A. Bulgac and Michael McNeil Forbes, Phys. Rev. Lett. 101, 215301 (2008).

[34] J. C. Pei, J. Dukelsky, and W. Nazarewicz, Phys. Rev. A 82, 021603(R) (2010).

[35] Michael McNeil Forbes, S. Gandolfi, and A. Gezerlis, Phys. Rev. Lett. 106, 235303 (2011).

[36] N. Navon, S. Nascimbène, F. Chevy, and C. Salomon, Science 328, 729 (2010).

[37] Michael McNeil Forbes, S. Gandolfi, and A. Gezerlis, Phys. Rev. A 86, 053603 (2012).

[38] M. Ku, A. T. Sommer, L. W. Cheuk, and M. W. Zwierlein, Science 335, 563 (2012).

[39] A. Schirotzek, Y.-i. Shin, C. H. Schunck, and W. Ketterle, Phys. Rev. Lett. 101, 140403 (2008).
[40] J. Braun, S. Diehl, and M. M. Scherer, Phys. Rev. A 84, 063616 (2011).

[41] J. Carlson and S. Reddy, Phys. Rev. Lett. 95, 060401 (2005).

[42] O. Juillet, New J. Phys. 9, 163 (2007).

[43] M. Kortelainen, N. Hinohara, and W. Nazarewicz, Phys. Rev. C 92, 051302(R) (2015).

[44] M. K. Tey, L. A. Sidorenkov, Edmundo R. Sánchez Guajardo, R. Grimm, M. J. H. Ku, M. W. Zwierlein, Y.-H. Hou, L. Pitaevskii, and S. Stringari, Phys. Rev. Lett. 110, 055303 (2013).

[45] A. Baran, A. Bulgac, Michael McNeil Forbes, G. Hagen, W. Nazarewicz, N. Schunck, and M. V. Stoitsov, Phys. Rev. C 78, 014318 (2008).

[46] P. Avogadro and C. A. Bertulani, Phys. Rev. C 88, 044319 (2013).

[47] S. Nascimbène, N. Navon, K. J. Jiang, L. Tarruell, M. Teichmann, J. McKeever, F. Chevy, and C. Salomon, Phys. Rev. Lett. 103, 170402 (2009).

[48] M. J. Wright, S. Riedl, A. Altmeyer, C. Kohstall, E. R. Sánchez Guajardo, J. Hecker Denschlag, and R. Grimm, Phys. Rev. Lett. 99, 150403 (2007).

[49] E. Khan, Phys. Rev. C 80, 011307(R) (2009).

[50] M. Matsuo, Nucl. Phys. A 696, 371 (2001).

[51] G. F. Bertsch and R. A. Broglia, Oscillations in Finite Quantum Systems (Cambridge University Press, Cambridge, U.K., 1994).

[52] T. Bergeman, M. G. Moore, and M. Olshanii, Phys. Rev. Lett. 91, 163201 (2003).

[53] X. J. Liu, H. Hu, and P. D. Drummond, Phys. Rev. A 76, 043605 (2007).

[54] A. A. Orel, P. Dyke, M. Delehaye, C. J. Vale, and H. Hu, New J. Phys. 13, 113032 (2011). 